\title{
Differential transcription of the major antigenic protein 1 multigene family of Ehrlichia ruminantium in Amblyomma variegatum ticks
}

\author{
M. Postigo ${ }^{\mathrm{a}, \mathrm{b}}$, A. Taoufik ${ }^{\mathrm{a}}$, L. Bell-Sakyi ${ }^{\mathrm{b}}$, E. de Vries ${ }^{\mathrm{a}}$, W.I. Morrison ${ }^{\mathrm{b}}$ and \\ F. Jongejan ${ }^{a, c}$
}

${ }^{a}$ Utrecht Centre for Tick-borne Diseases, Faculty of Veterinary Medicine, Utrecht University, P.O. Box 80165, 3508 TD Utrecht, The Netherlands

${ }^{b}$ Centre for Tropical Veterinary Medicine, Royal (Dick) School of Veterinary Studies, University of Edinburgh, Easter Bush, Roslin, Midlothian EH25 9RG, Scotland, UK ${ }^{c}$ Department of Veterinary Tropical Diseases, Faculty of Veterinary Science, University of Pretoria, 0110 Onderstepoort, South Africa

\section{Abstract}

The rickettsial pathogen Ehrlichia ruminantium causes heartwater in ruminants and is transmitted by ticks of the genus Amblyomma. The map1 gene, encoding the major surface protein MAP1, is a member of a multigene family containing 16 paralogs. In order to investigate differential transcription of genes of the map1 multigene family in vivo in unfed and feeding ticks, RNA was extracted from midguts and salivary glands of E. ruminantium-infected adult female Amblyomma variegatum ticks and analysed by RTPCR using MAP1 paralog-specific primers. In unfed ticks, only transcripts from the map1-1 gene were observed in midguts and no transcripts were detected in salivary glands. In feeding ticks, map1-1 transcripts were more abundant in midguts whereas high levels of map1 transcripts were observed in salivary glands. Our results show that differential transcription of genes of the E. ruminantium map1 cluster occurs in vivo in different tissues of infected ticks before and during transmission feeding, indicating that 
this multigene family may be involved in functions of biological relevance in different stages of the life cycle of E. ruminantium.

\section{Article Outline}

1. Introduction

2. Materials and methods

2.1. Laboratory propagation of ticks

2.2. Experimental infection of sheep and ticks

2.3. Growth and harvest of E. ruminantium from in vitro cell cultures

2.4. DNA and RNA isolation

2.5. Real-time PCR

2.6. cDNA synthesis and paralog-specific PCR

3. Results

3.1. Experimental infection of sheep and ticks

3.2. Real-time PCR

3.3. Transcriptional analysis

4. Discussion

Acknowledgements

References

\section{Introduction}

The rickettsial pathogen Ehrlichia ruminantium is the causative agent of heartwater or cowdriosis. The disease affects domestic and wild ruminants and occurs throughout subSaharan Africa and also on some islands in the Caribbean (Uilenberg, 1983). The organism is transstadially transmitted by ticks of the genus Amblyomma (Bezuidenhout, 1987).

Multigene families encoding for outer membrane proteins have been identified in the genus Ehrlichia and designated P28 (OMP-1) in Ehrlichia chaffeensis, P30 in Ehrlichia canis and the major antigenic protein 1 (MAP1) family in E. ruminantium (Ohashi et al., 
1998a, Ohashi et al., 1998b and Sulsona et al., 1999). These genes are thought to be responsible for adaptation to the environment in the tick and involved in transmission to the mammalian host (Felek et al., 2003 and Unver et al., 2002).

Recently, the entire map1 cluster of the Welgevonden isolate of E. ruminantium was characterised and shown to contain 16 genes located upstream of a secA gene (van Heerden et al., 2004). All 16 paralogs were transcriptionally active when E. ruminantium was grown in bovine endothelial cells, whereas between 4 and 11 paralogs were found to be transcribed in E. ruminantium-infected tick cell lines (Bekker et al., 2005; van Heerden et al., 2004) and transcripts of only two out of three paralogs were detected in unfed infected adult Amblyomma variegatum ticks (Bekker et al., 2002). In the present study, we analysed transcription of the entire map1 cluster in midguts and salivary glands of E. ruminantium-infected Amblyomma variegatum ticks and assessed levels of transcription of map1 genes in relation to the numbers of organisms present in different tissues of unfed and feeding ticks.

\section{Materials and methods}

\subsection{Laboratory propagation of ticks}

Amblyomma variegatum were obtained from the Centre de Coopération Internationale en Recherche Agronomique pour le Développement (CIRAD-EMVT), Pointe-à-Pitre, Guadeloupe, and established as an E. ruminantium-free laboratory colony at Utrecht University. Larvae and nymphs were fed on rabbits, and adults were fed on sheep. The engorged, moulting and unfed stages were maintained at $27{ }^{\circ} \mathrm{C}, 95 \%$ relative humidity (Heyne et al., 1987).

\subsection{Experimental infection of sheep and ticks}

Naïve female Texelaar sheep, aged between 6 and 8 months, were maintained at Utrecht University under the regulations of the Animal Ethics Committee (Dierexperimentencommissie) of the University. The sheep were monitored daily for changes in rectal temperature and clinical signs of disease, and serum samples were collected weekly. Sheep \# 3464 was inoculated intravenously with $2 \mathrm{ml}$ of cryopreserved sheep blood infected with the Gardel isolate of E. ruminantium (Uilenberg et al., 1985) 
(designated stabilate CR366 at Utrecht University). Uninfected Amblyomma variegatum nymphs were applied in cloth patches onto the back of the infected sheep on days 7, 8, 9 and 10 after inoculation and allowed to acquisition-feed during the febrile reaction of the animal. Engorged nymphs were collected and allowed to moult and harden at $27^{\circ} \mathrm{C}, 95 \%$ relative humidity for 6-8 weeks. Approximately 50 each of the resultant adult males and females were transmission-fed on the back of a second susceptible sheep \# 3456.

Following attachment, five females were detached daily on days $1-4$ of feeding. Midguts and salivary gland pairs from five unfed ticks and from five ticks harvested on each day of feeding were dissected out and pooled according to tissue and feeding day. Tissues from uninfected control ticks, both unfed and fed on an uninfected sheep for 1-4 days, were processed similarly.

\subsection{Growth and harvest of $\boldsymbol{E}$. ruminantium from in vitro cell cultures}

The Gardel isolate of E. ruminantium was cultured in bovine umbilical cord endothelial cells (BUE) as described previously (Jongejan et al., 1991). Bacterial growth was monitored by microscopic examination of Giemsa-stained cytocentrifuge smears. When E. ruminantium-infected BUE cultures showed about $90 \%$ of cytolysis due to infection, the remaining adherent cells were scraped from the bottom of the culture flasks and the cultures centrifuged at $15,000 \times g$ for $20 \mathrm{~min}$ at $4{ }^{\circ} \mathrm{C}$. The resultant pellet containing both infected cells and free E. ruminantium organisms was resuspended in $1 \mathrm{ml}$ of Tri reagent (Sigma) and frozen at $-70^{\circ} \mathrm{C}$ until used for DNA and RNA extraction.

\subsection{DNA and RNA isolation}

The midgut and salivary gland pools dissected from the unfed ticks and ticks harvested daily after 1-4 days of feeding either on sheep \# 3456 (E. ruminantium-infected ticks), or on the uninfected control sheep (uninfected control ticks), were snap-frozen in liquid nitrogen and ground into powder. DNA and RNA from the resultant homogenised tissues were simultaneously extracted using the TRI reagent protocol (Sigma) and cleaned using the columns from QIAamp extraction kits (Qiagen) according to the manufacturer's instructions. DNA and RNA were eluted from columns with $100 \mu$ l of elution buffer (10 mM Tris/HCl; $0.5 \mathrm{mM}$ EDTA; $\mathrm{pH} 9.0$ ) or $50 \mu 1$ of RNase-free water, respectively. 
Total DNA and RNA from E. ruminantium-infected endothelial cell cultures were extracted as described above and 10 -fold dilution series $\left(10^{0}\right.$ to $\left.10^{-3}\right)$ were prepared from these original DNA and RNA stocks diluted in RNase-free water.

\subsection{Real-time PCR}

The map1-1 gene (GenBank accession no. AY652746) was selected as the target to quantify bacterial DNA in tick tissues and in the 10-fold DNA dilution series from $E$. ruminantium-infected in vitro cultures. Primers specific for the map1-1 gene (forward primer F3: 5'-TAC GGT AAA GAC TCT CCA ACT AAA-3'; reverse primer R1: 5'TAT GCA GCT TCA ATC TCT ACT-3') were used to amplify a fragment of $182 \mathrm{bp}$ from test DNA samples and from a positive control plasmid, PBAD/Myc-His (Invitrogen), containing the map1-1 gene. The PCR reaction mixture used was as follows: $12.5 \mu \mathrm{l}$ of SYBR Green supermix (Bio-Rad), $1 \mu \mathrm{l}$ of forward primer, $1 \mu \mathrm{l}$ of reverse

primer $\left(0.4 \mu \mathrm{mol}^{-1}\right.$ final concentration) and $5 \mu \mathrm{l}$ of template, in a $25 \mu \mathrm{l}$ PCR reaction. The cycling programme consisted of: $95^{\circ} \mathrm{C}$ for $15 \mathrm{~min}$; and cycles of denaturing, $95^{\circ} \mathrm{C}$ for $30 \mathrm{~s}$; annealing, $55^{\circ} \mathrm{C}$ for $30 \mathrm{~s}$; and extension, $72{ }^{\circ} \mathrm{C}$ for $30 \mathrm{~s}$, repeated 40 times. $\mathrm{Ct}$ values were determined and melting curve graphs analysed accordingly using the iCycler software.

\section{6. cDNA synthesis and paralog-specific PCR}

Reverse transcriptase PCR (RT-PCR) was carried out on RNA from E. ruminantiuminfected and uninfected ticks and from the 10-fold RNA dilution series extracted from in vitro cultures. Five microlitres of DNase-treated RNA was used to prepare $20 \mu \mathrm{l}$ of cDNA using $100 \mathrm{ng}$ of random hexamer primers of the SuperScript ${ }^{\mathrm{TM}}$ first-strand synthesis system (Invitrogen) according to the manufacturer's instructions. Control samples were prepared by omitting reverse transcriptase from the reaction. Two microlitres of cDNA was used subsequently as template in a PCR reaction containing: 1X Taq PCR buffer (Promega), $3 \mathrm{mM} \mathrm{MgCl}, 1.25 \mathrm{U}$ of Taq polymerase (Promega), $400 \mu \mathrm{M}$ of each deoxynucleoside triphosphate and 10 pmol of each primer in a $25 \mu \mathrm{l}$ reaction. The sequences of the paralog-specific primers used were those described previously (van Heerden et al., 2004). Reactions were carried out in an iCycler (Bio-Rad) using the 
following programme: $2 \mathrm{~min}$ at $94{ }^{\circ} \mathrm{C}$ followed by 40 cycles of $30 \mathrm{~s}$ at $94{ }^{\circ} \mathrm{C}, 30 \mathrm{~s}$ at $55^{\circ} \mathrm{C}, 1 \mathrm{~min}$ at $72{ }^{\circ} \mathrm{C}$ and a final elongation step of $7 \mathrm{~min}$ at $72{ }^{\circ} \mathrm{C}$. Positive controls incorporated genomic DNA and cDNA from E. ruminantium-infected endothelial cell cultures. PCR products were visualised by running the samples on agarose gels and staining with ethidium bromide.

\section{Results}

\subsection{Experimental infection of sheep and ticks}

Sheep \# 3464, infected by injection with E. ruminantium blood stabilate CR366, reacted with fever 13 days post inoculation and treatment with $10 \%$ engemycin was started on the third consecutive day of fever; however the sheep died on day 17 post inoculation. Sheep \# 3456, infected by tick bite, developed fever 13 days after tick application and was found dead on day 14. Heartwater was confirmed in both sheep post mortem by observation of E. ruminantium colonies in Giemsa-stained brain smears. Pools of dissected midguts and salivary glands from five unfed female ticks and five female ticks harvested daily after 1-4 days of feeding were submitted to simultaneous DNA and RNA extraction. To assess if the samples were infected with E. ruminantium, a PCR was run on genomic DNA using two different combinations of primers specific to the map1-1 gene. Amplicons of the corresponding expected sizes were observed in all samples of midguts and salivary glands from unfed and feeding ticks indicating that all the pools analysed were positive for E. ruminantium infection (data not shown). No products were obtained from uninfected ticks using the same PCR conditions (data not shown), confirming specificity of the primers.

\subsection{Real-time PCR}

In order to quantify the number of bacteria present in the samples, real-time PCR was performed on DNA extracted from pools of midguts and salivary glands as well as from E. ruminantium-infected endothelial cell cultures. Quantitative measurements were based on a standard plot using a 10-fold dilution series of a plasmid encoding the map1-1 gene of E. ruminantium. Since map1-1 is known to be a single copy gene (Collins et al., 2005), it allows a direct estimation of the number of E. ruminantium organisms per sample. 
Standards were diluted in water, as amplification of plasmid standards diluted in carrier DNA (DNA from uninfected ticks) did not interfere with the sensitivity of the test (data not shown). A linear correlation was found within the range of $10^{2}-10^{6}$ plasmid copies per PCR reaction and comparable standard curve slopes were obtained from two independent experiments. An identical melting temperature of $77.5^{\circ} \mathrm{C}$ was obtained for the standard plasmids and all samples analysed containing E. ruminantium template DNA. There was no interference in the PCR reaction from non-specific products or primer dimers (data not shown). The results are shown in Fig. 1a and are expressed as the number of bacteria per tick (equivalent to number of bacteria per $20 \mu \mathrm{l}$ of DNA suspension). E. ruminantium was present in both midguts and salivary glands of unfed infected ticks (day 0 ). The numbers of bacteria in midguts increased seven-fold from $1.2 \times 10^{6}$ per tick in unfed ticks to $9.3 \times 10^{6}$ per tick after 3 days of feeding. The bacterial load in salivary glands increased 40 -fold from $1.2 \times 10^{5}$ per tick in unfed ticks to $5.3 \times 10^{6}$ per tick after 3 days of feeding. In salivary glands a four-fold decrease in numbers of bacteria was observed after day 3. In the serial dilutions of DNA from $E$. ruminantium-infected endothelial cells, the number of bacteria per $20 \mu$ of DNA suspension ranged from $10^{7}$ bacteria in the undiluted sample to $10^{4}$ bacteria in the $10^{-3}$ dilution.

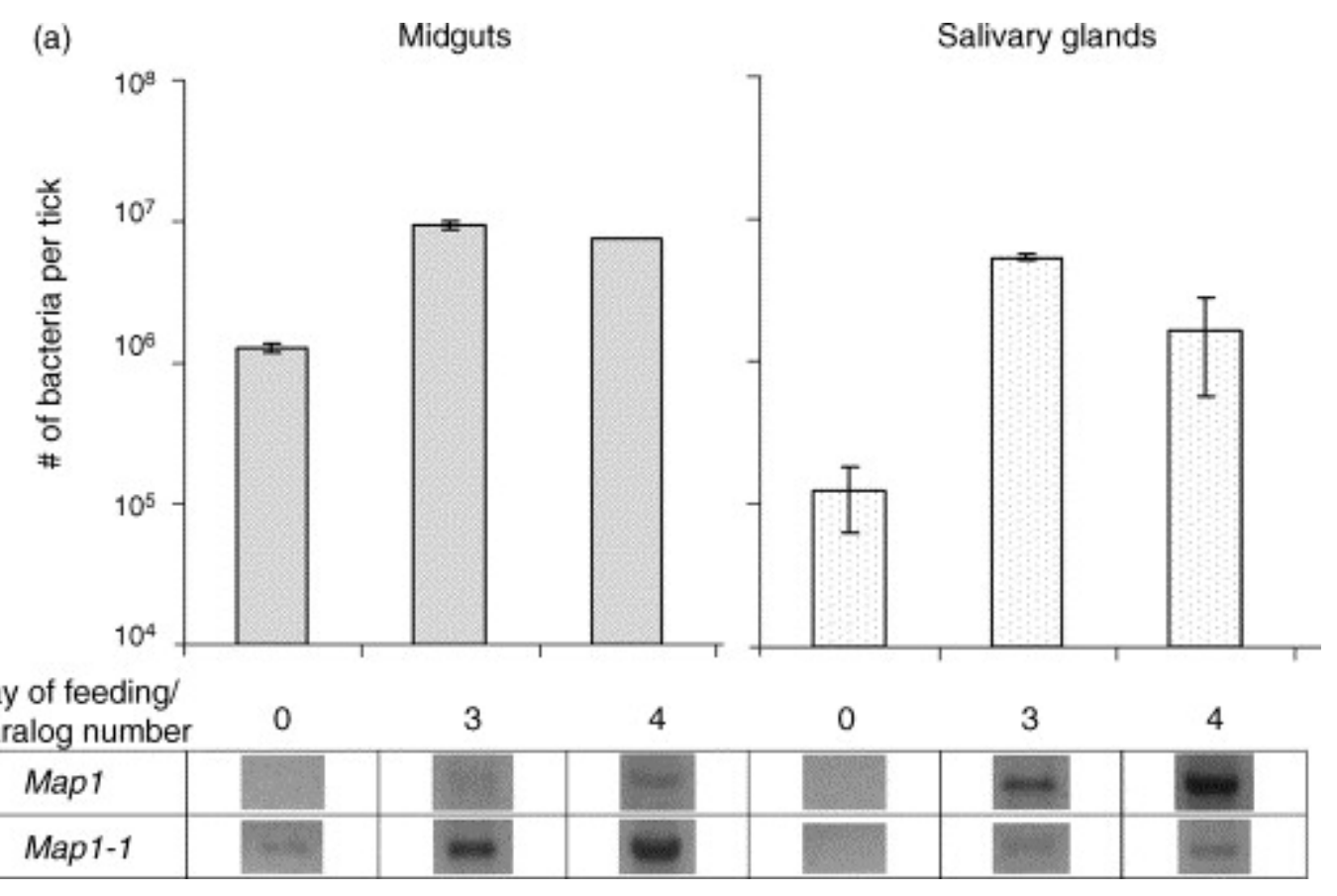

(b) Day of feeding/
Paralog number 0
\begin{tabular}{|c|l|l|l|l|l|l|l|l|}
\hline Map1 & & & & & & & & \\
\hline Map1-1 & & & & & & & & \\
\hline
\end{tabular}


Fig. 1. Numbers of E. ruminantium organisms and transcription of map1 and map1-1 genes in midguts and salivary glands of unfed ticks (day 0 ) and ticks fed for 3 and 4 days. DNA and RNA were simultaneously extracted from pools of five midguts and five salivary gland pairs per feeding day. (a) Bacterial numbers were determined by real-time PCR and are presented as mean per tick \pm standard deviation. The mean values were calculated from three readings obtained from two different experiments on the same set of samples for each time point. (b) The panels show ethidium bromide-stained RT-PCR products of E. ruminantium map1 and map1-1 paralogs from midguts and salivary glands of unfed and feeding ticks, allowing comparison between amount of RT-PCR products and the number of bacteria present on each day in the respective tissue.

\subsection{Transcriptional analysis}

RT-PCR was performed to study the transcriptional activity of the entire map1 cluster in vivo in ticks and to assess possible differences in transcription of paralogs of the map1 cluster between midguts and salivary glands of unfed and feeding ticks and between different days of feeding.

Typical transcriptional profiles of the E. ruminantium map1 cluster in ticks are shown in Fig. 2. In unfed ticks, only amplicons from the map1-1 gene were detected in midguts (Fig. 2c and d). In contrast in feeding ticks (day 4 in Fig. 2e and f), PCR products were detected in midguts and salivary glands for both the map1 (685 bp) and map1-1 (550 bp) genes. Additional bands, which did not correspond to the expected molecular mass, were seen when using map1-4, 1-7, 1-9 and 1-10 gene-specific primers (Fig. 2). Sequencing of all amplicons demonstrated that only the transcripts from the map1 and map1-1 genespecific primers were E. ruminantium-specific products. The expected PCR products were obtained from E. ruminantium positive control DNA and cDNA samples using each primer pair, confirming the ability of the primers to amplify the target sequences (Fig. 2a and b). No transcripts of any of the map1 paralogs were detected in uninfected tick tissue or in infected tick tissue without reverse transcriptase, indicating the absence of contamination with genomic DNA in the RNA preparation (data not shown). 


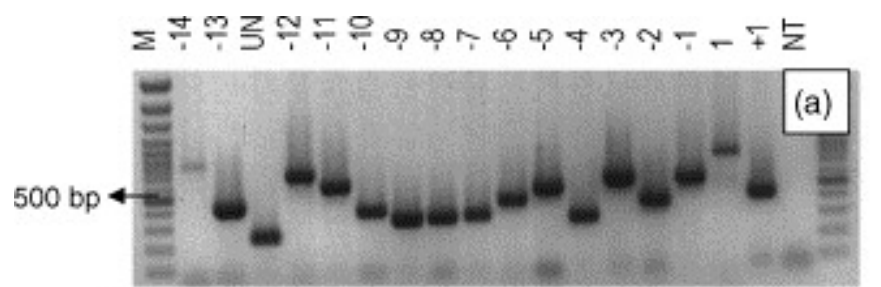

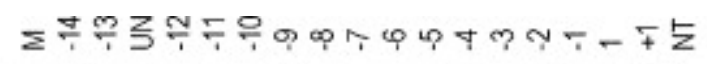
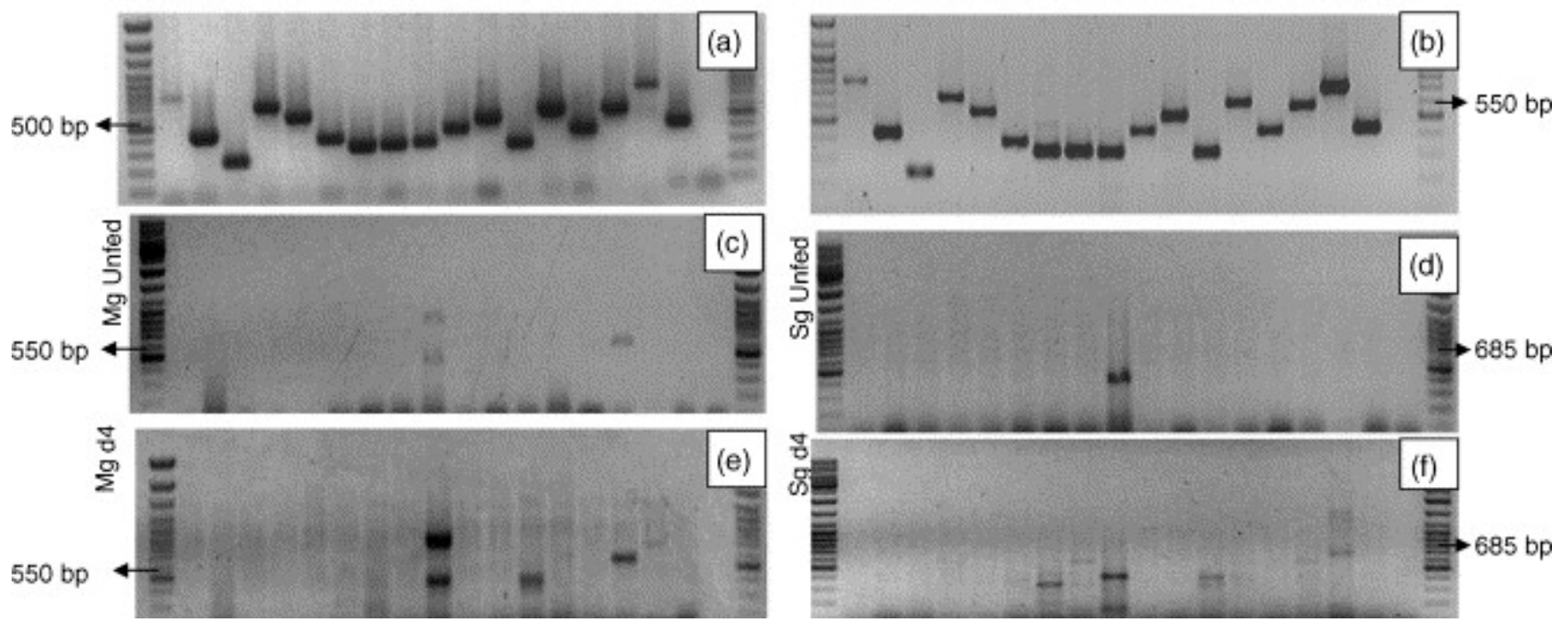

Fig. 2. Transcriptional profiles of the E. ruminantium map1 cluster in unfed and feeding ticks. The panels show PCR products from genomic DNA (a) and cDNA (b) amplified with map1 cluster primers for [left to right] map1-14 to map1 +1 ; $\mathrm{UN}$ is an unknown gene (van Heerden et al., 2004); (c and d) RT-PCR products from E. ruminantiuminfected midguts and salivary glands respectively of unfed ticks and (e and f) from midguts and salivary glands respectively of ticks that had fed for 4 days. M: molecular marker, the band that corresponds to $500 \mathrm{bp}$ is indicated in panel a. NT: no template. Nucleotide base pair numbers of specific amplified products corresponding to map1-1 (550 bp) and map1 (685 bp) are indicated on the left and right panels, respectively. Gels were stained with ethidium bromide.

Transcription of map1 and map1-1 in different tissues and on different days of feeding was compared with numbers of bacteria (Fig. 1a and b). Transcripts of the map1-1 gene were found in midguts of unfed and feeding ticks, whereas transcripts of the map1 gene were detected in midguts of ticks after 4 days of feeding. The amount of the amplified map1-1 gene product increased as feeding progressed despite a reduction of the number of bacteria on day 4 . In contrast, in salivary glands no transcripts were found in unfed ticks, whereas in feeding ticks transcripts of the map1 and map1-1 genes were detected on days 3 and 4 of feeding, with the map1 transcripts being predominant. The highest signal generated from the amplified map1 gene product was observed on day 4 despite a 
significant reduction in the number of bacteria on that day. These results suggest that there is differential transcription of map1 and map1-1 genes depending on the stage of feeding of the tick and the location of the bacteria within the tick. The amount of RNA extracted from the tick tissues was insufficient for quantitative RT-PCR analysis so, for comparison purposes, transcriptional analysis of the map1 and map1-1 genes was carried out on the dilution series prepared from the RNA extracted from the E. ruminantiuminfected endothelial cells. Transcripts of the map1 gene were detected in all the dilutions, which had contained RNA from between $10^{7}$ and $10^{4}$ bacteria, whereas transcripts of the map1-1 gene were detected only in the $10^{0}$ and $10^{-1}$ dilutions of RNA extracted from $10^{7}$ and $10^{6}$ bacteria, respectively; the numbers of bacteria determined by real-time PCR in the tick tissues fell within this range. For both genes, the intensity of the bands diminished as the number of bacteria decreased (Fig. 3).

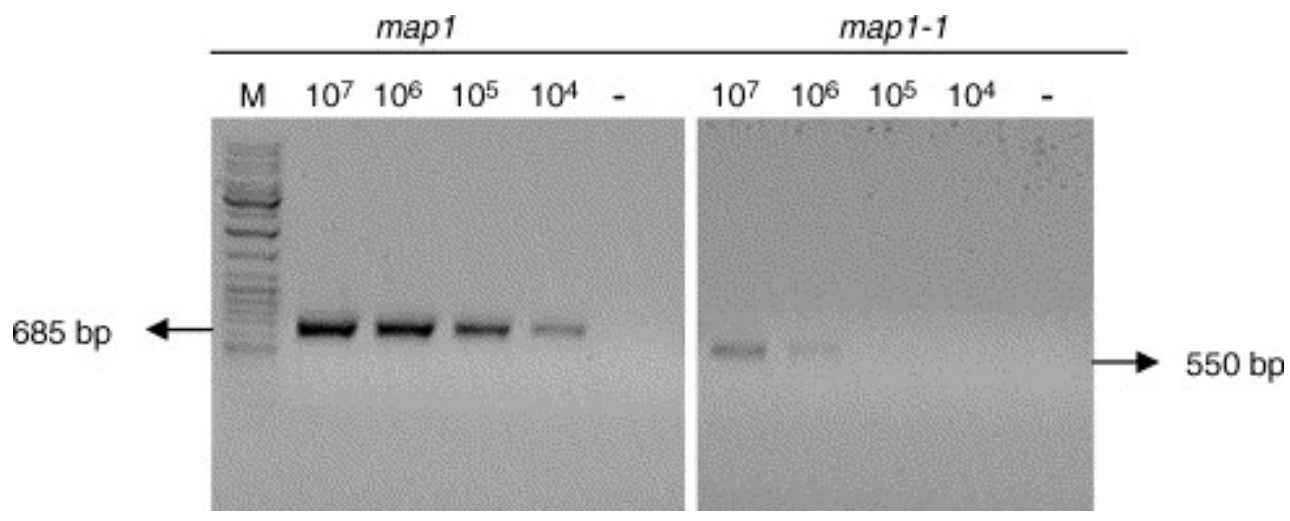

Fig. 3. Transcription of map1 and map1-1 genes in E. ruminantium-infected endothelial cell cultures. DNA and RNA were simultaneously extracted from E. ruminantium (Gardel)-infected endothelial cells. Numbers of bacteria, quantified by real-time PCR in a serial dilution of DNA, are shown at the top of both panels. mRNA generated by the respective number of bacteria, was converted to cDNA and amplified using specific primers for the map1 (left panel) and map1-1 (right panel) genes. RT-PCR products were visualised on agarose gels stained with ethidium bromide. 


\section{Discussion}

In this study, we analysed the transcriptional activity of the map1 multigene family in different tissues of E. ruminantium-infected ticks and found differences in transcription between midguts and salivary glands of both unfed and feeding ticks.

Out of 16 paralogs examined, only transcripts from two genes, map1 and map1-1, were detected in ticks. This was in agreement with a preliminary study (Bekker et al., 2002) in which transcripts of map1 and map1-1, but not map1-2, were detected in whole unfed adult Amblyomma variegatum ticks infected with E. ruminantium (Senegal). In the closely-related pathogens E. canis and E. chaffeensis, transcription of only one paralog each of the orthologous multigene families was detected in vector ticks (Unver et al., 2001 and Unver et al., 2002); in both cases the transcribed gene was the ortholog of map1-1. In the present study, map1-1 transcripts were detected in unfed ticks only in midguts, despite the presence of E. ruminantium organisms in the salivary glands before the start of feeding as determined by real-time PCR. During tick feeding, transcripts of the map1 paralog were more abundant in salivary glands than in midguts, although the number of bacteria was always substantially higher in midguts than in salivary glands. Moreover, the number of bacteria in both tissues showed a peak on day 3 while levels of map1-1 and map1 transcripts increased over the time course of tick feeding in midguts and salivary glands, respectively. In contrast, in the 10-fold dilution series prepared from E. ruminantium grown in endothelial cells, transcripts of the map1 and map1-1 genes decreased as the number of bacteria decreased. These observations indicate that the transcription pattern observed in infected ticks reflected real differences in the levels of mRNA rather than in the numbers of bacteria, and that differential regulation of these genes occurs in ticks, related to the tissue and the stage of feeding. Our results are similar to those reported for differential transcription of members of the $p 44$ multigene family of Anaplasma phagocytophilum during tick transmission (IJdo et al., 2002). Several p44 transcripts were detected in salivary glands of transmission-feeding nymphs during engorgement but not in unfed nymphs, suggesting that tick engorgement may induce transcription of $p 44$ genes.

The development of E. ruminantium in ticks and the route of transmission to the mammalian host are incompletely understood. Electron microscopy studies detected 
colonies of E. ruminantium in sections of midgut epithelial cells of unfed infected nymphal Amblyomma hebraeum (infected as larvae), as well as in those collected on days 1-4 of feeding, while in salivary glands colonies of bacteria were only found in nymphs that had fed for 4 days (Kocan et al., 1987a). E. ruminantium colonies were detected in midguts of adult Amblyomma hebraeum on days 1-3 of transmission-feeding, but not thereafter and not in the corresponding salivary glands (Hart et al., 1991), and in midguts of moulting nymphal and newly moulted adult Amblyomma variegatum (Kocan et al., 1987b). An E. ruminantium-specific DNA probe has been used to detect E. ruminantium infection in midguts and salivary glands of unfed adult Amblyomma hebraeum (Yunker et al., 1993) and midguts of unfed adult Amblyomma variegatum incubated for $24 \mathrm{~h}$ at $37^{\circ} \mathrm{C}$ (Waghela et al., 1991). To our knowledge, the present study is the first report of quantification of E. ruminantium in midguts and salivary glands of unfed and feeding ticks. Our results from real-time PCR confirmed that E. ruminantium was already present in both midguts and salivary glands of infected ticks before the start of the transmission feeding and that the number of bacteria increased substantially after 3 days of feeding in both tissues. The midguts appeared to be the main tissue location for colonisation and replication of E. ruminantium as the number of bacteria always remained higher in midguts than in salivary glands, consistent with the relative ease of detection by previous workers of colonies in midguts compared to salivary glands (Cowdry, 1925, Kocan et al., 1987a and Hart et al., 1991). The presence of mammal-infective E. ruminantium in tick saliva has not been reliably demonstrated (Bezuidenhout, 1981), but our results suggest that transmission of organisms replicating in the salivary glands may be the principal route of infection for E. ruminantium as there was a dramatic decrease in the number of bacteria in salivary glands, but not in midguts, between days 3 and 4 . This observation is consistent with the finding that $E$. ruminantium requires a period of about $75 \mathrm{~h}$ of feeding prior to transmission by adult ticks (Bezuidenhout, 1987).

A marked increase in the infectivity of E. ruminantium-infected ticks during feeding has been reported (Bezuidenhout, 1981), which may reflect replication leading to a higher number of organisms and/or developmental maturation into infective stages. Reticulated bodies and, to a lesser extent, electron-dense forms, which could be considered to resemble respectively the non-infectious forms and the infective stages (elementary 
bodies) of the bacteria occurring in mammalian cell cultures (Jongejan et al., 1991), have been detected in both midguts and salivary glands of partially fed infected Amblyomma hebraeum ticks by electron microscopy (Kocan et al., 1987a). The differentiation of $E$. ruminantium to mammalian-infective forms in the tick appears to be a complex process in which the differential transcription of genes coding for outer membrane proteins of $E$. ruminantium in unfed and feeding ticks as demonstrated here for two map1 gene family paralogs may be involved. However it will be necessary to determine whether the differential transcription of map1 paralogs observed in vitro (Bekker et al., 2005) and in vivo is reflected at the protein level.

\section{References}

Bekker et al., 2002 C.P.J. Bekker, L. Bell-Sakyi, E.A. Paxton, D. Martinez, A. Bensaid and F. Jongejan, Transcriptional analysis of the major antigenic protein 1 multigene family of Cowdria ruminantium, Gene 285 (2002), pp. 193-201.

Bekker et al., 2005 C.P.J. Bekker, M. Postigo, A. Taoufik, L. Bell-Sakyi, C. Ferraz, D. Martinez and F. Jongejan, Transcription analysis of the major antigenic protein 1 multigene family of three in vitro-cultured Ehrlichia ruminantium isolates, J. Bacteriol. 187 (2005), pp. 4782-4791.

Bezuidenhout, 1981 J.D. Bezuidenhout, The development of a new heartwater vaccine, using Amblyomma hebraeum nymphae infected with Cowdria ruminantium. In: G.B. Whitehead and J.D. Gibson, Editors, Tick Biology and Control, Tick Research Unit, Grahamstown, South Africa (1981), pp. 41-45.

Bezuidenhout, 1987 J.D. Bezuidenhout, Natural transmission of heartwater, Onderstepoort J. Vet. Res. 54 (1987), pp. 349-351.

Cowdry, 1925 E.V. Cowdry, Studies on the etiology of heartwater II. Rickettsia ruminantium (n.sp.) in the tissues of ticks transmitting the disease, J. Exp. Med. 42 (1925), pp. 253-274.

Collins et al., 2005 N.E. Collins, J. Liebenberg, E.P. De Villiers, K.A. Brayton, E. Louw, A. Pretorius, F.E. Faber, H. van Heerden, A. Josemans, M. van Kleef, H.C. Steyn, M.F. van Strijp, E. Zweygarth, F. Jongejan, J.C. Maillard, D. Berthier, M. Botha, F. Joubert, C.H. Corton, N.R. Thomson, M.T. Allsopp and B.A. Allsopp, The genome of the 
heartwater agent Ehrlichia ruminantium contains multiple tandem repeats of actively variable copy number, PNAS 102 (2005), pp. 838-843.

Felek et al., 2003 S. Felek, R. Greene and Y. Rikihisa, Transcriptional analysis of $p 30$ major outer membrane protein genes of Ehrlichia canis in naturally infected ticks and sequence analysis of p30-10 of E. canis from diverse geographic regions, J. Clin.

Microbiol. 41 (2003), pp. 886-888.

Hart et al., 1991 A. Hart, K.M. Kocan, J.D. Bezuidenhout and L. Prozesky, Ultrastructural morphology of Cowdria ruminantium in midgut epithelial cells of adult Amblyomma hebraeum female ticks, Onderstepoort J. Vet. Res. 58 (1991), pp. 187-193. Heyne et al., 1987 W. Heyne, E.G. Elliot and J.D. Bezuidenhout, Rearing and infection techniques for Amblyomma species to be used in heartwater transmission experiments, Onderstepoort J. Vet. Res. 54 (1987), pp. 461-471.

IJdo et al., 2002 J.W. IJdo, C. Wu, S.R. Telford and E. Fikrig, Differential expression of the $p 44$ gene family in the agent of human granulocytic ehrlichiosis, Infect. Immun. 70 (2002), pp. 5295-5298.

Jongejan et al., 1991 F. Jongejan, T.A. Zandbergen, P.A. van de Wiel, M. de Groot and G. Uilenberg, The tick-borne rickettsia Cowdria ruminantium has a Chlamydia-like developmental cycle, Onderstepoort J. Vet. Res. 58 (1991), pp. 227-237.

Kocan et al., 1987a K.M. Kocan, J.D. Bezuidenhout and A. Hart, Ultrastructural features of Cowdria ruminantium in midgut epithelial cells and salivary glands of nymphal Amblyomma hebraeum, Onderstepoort J. Vet. Res. 54 (1987), pp. 87-93.

Kocan et al., 1987b K.M. Kocan, S.P. Morzaria, W.P. Voigt, J. Kiarie and A.D. Irvin, Demonstration of colonies of Cowdria ruminantium in midgut epithelial cells of Amblyomma variegatum, Am. J. Vet. Res. 48 (1987), pp. 356-360.

Ohashi et al., 1998a N. Ohashi, N. Zhi, Y. Zhang and Y. Rikihisa, Immunodominant major outer membrane proteins of Ehrlichia chaffeensis are encoded by a polymorphic multigene family, Infect. Immun. 66 (1998), pp. 132-139.

Ohashi et al., 1998b N. Ohashi, A. Unver, N. Zhi and Y. Rikihisa, Cloning and characterization of multigenes encoding the immunodominant 30-kilodalton major outer membrane proteins of Ehrlichia canis and application of the recombinant protein for serodiagnosis, J. Clin. Microbiol. 36 (1998), pp. 2671-2680. 
Sulsona et al., 1999 C.R. Sulsona, S.M. Mahan and A.F. Barbet, The map1 gene of Cowdria ruminantium is a member of a multigene family containing both conserved and variable genes, Biochem. Biophys. Res. Commun. 257 (1999), pp. 300-305.

Uilenberg, 1983 G. Uilenberg, Heartwater (Cowdria ruminantium infection): current status, Adv. Vet. Sci. Comp. Med. 27 (1983), pp. 427-480.

Uilenberg et al., 1985 G. Uilenberg, E. Camus and N. Barré, Quelques observations sur une souche de Cowdria ruminantium isolée en Guadeloupe (Antilles francaises), Rev. Elev. Med. Vet. Pays Trop. 38 (1985), pp. 34-42.

Unver et al., 2001 A. Unver, N. Ohashi, T. Tajima, R.W. Stich, D. Grover and Y. Rikihisa, Transcriptional analysis of p30 major outer membrane multigene family of Ehrlichia canis in dogs, ticks, and cell culture at different temperatures, Infect. Immun. 69 (2001), pp. 6172-6178.

Unver et al., 2002 A. Unver, Y. Rikihisa, R.W. Stich, N. Ohashi and S. Felek, The omp-1 major outer membrane multigene family of Ehrlichia chaffeensis is differentially expressed in canine and tick hosts, Infect. Immun. 70 (2002), pp. 4701-4704. van Heerden et al., 2004 H. van Heerden, N.E. Collins, K.A. Brayton, C. Rademeyer and B.A. Allsopp, Characterization of a major outer membrane protein multigene family in Ehrlichia ruminantium, Gene 330 (2004), pp. 159-168.

Waghela et al., 1991 S.D. Waghela, F.R. Rurangirwa, S.M. Mahan, C.E. Yunker, T.B. Crawford, A.F. Barbet, M.J. Burridge and T.C. McGuire, A cloned DNA probe identifies Cowdria ruminantium in Amblyomma variegatum ticks, J. Clin. Microbiol. 29 (1991), pp. 2571-2577.

Yunker et al., 1993 C.E. Yunker, S.M. Mahan, S.D. Waghela, T.C. McGuire, F.R. Rurangirwa, A.F. Barbet and L.A. Wassink, Detection of Cowdria ruminantium by means of a DNA probe, pCS20 in infected bont ticks, Amblyomma hebraeum, the major vector of heartwater in southern Africa, Epidemiol. Infect. 110 (1993), pp. 95-104.

Corresponding author at: Utrecht Centre for Tick-borne Diseases, Faculty of Veterinary Medicine, Utrecht University, P.O. Box 80165, 3508 TD Utrecht, The Netherlands. Tel.: +3130 2536932; fax: +31302532333. 